\title{
POLAR SORT: COMBINING MULTIDIMENSIONAL SCALING AND POLAR COORDINATES FOR MATRIX REORDERING
}

\author{
Celmar Guimarães da Silva \\ School of Technology, University of Campinas, R. Paschoal Marmo, 1888. Jardim Nova Itália. Limeira, SP, Brazil
}

\begin{abstract}
Matrix reordering algorithms aim to permute rows and columns of a matrix (or a matrix-based visualization, such as a heatmap) in order to reveal data patterns that could not be perceivable in some orderings of its columns and rows. Finding a good permutation, according to some evaluation criteria, is not straightforward, and many approaches try to find out a good tradeoff between algorithm execution time and output quality. This work argues that using multidimensional scaling and polar coordinates helps to find row and column orderings that reveal Band and Circumplex patterns if they are present in some permutation of a matrix. The proposed $\mathrm{O}\left(n^{3}\right)$ method - Polar Sort - uses classical MDS to find an initial 2-dimensional projection of rows (or columns) of the input matrix. After that, the method sorts the projected points according to their angular coefficients in a polar coordinate system whose center is the barycenter of these points. The algorithm then replicates this order to the correspondent rows (or columns). Experiments with synthetic data indicates that Polar Sort produces high-quality results for uncovering Band and Circumplex patterns, and that its mean execution time is as fast as the fastest tested methods for matrices with size $200 \times 200$. This paper also presents real-world examples in which Polar Sort shows those patterns.
\end{abstract}

\section{KEYWORDS}

Heatmaps, Information Visualization, Visualization Design and Evaluation Methods

\section{INTRODUCTION}

Matrix reordering algorithms (also called seriation algorithms) tries to permute rows and columns of a matrix in order to enable users to have a better comprehension about the data it contains, when it is presented as a visual structure (such as a heatmap, a Table Lens display etc.). A general principle is that similar rows (or columns) should be closer. Researchers have been trying many approaches to solve matrix reordering problems, such as dimensional reduction and spectral approaches (Behrisch et al., 2016); and some of them focus on unveiling specific sets of patterns (Rocha et al., 2017; Medina et al., 2016). In this work, we prove the Wilkinson's untested hypothesis (Wilkinson, 2005) that doing a bidimensional projection of rows and columns of a reorderable matrix, and ordering them according to the angles of their projected points in a polar coordinate system would be able to reveal Band and Circumplex patterns. We formalize and implement his idea as a matrix reordering algorithm called Polar Sort.

We used Matrix Reordering Analyzer tool (Silva et al., 2014) to compare Polar Sort to other algorithms that produce good results (in terms of output quality and execution time) regarding those focus patterns. We conclude that the proposed algorithm recompose those patterns even when the tested matrices have high noise ratios. Besides, its execution time is as fast as the ones from the other algorithms that reveal these patterns, for the tested scenarios.

We organize this paper as follows. Section 1 presents theoretical background about matrix patterns and matrix evaluation functions. Section 2 briefly presents other related reordering algorithms. Section 3 defines Polar Sort and exemplifies its outputs for the mentioned patterns. Section 4 evaluates our algorithm with synthetic data, compares its asymptotic complexity with other algorithms, and illustrates its output for real-world data. Section 5 presents conclusions and future work. 


\section{THEORETICAL BACKGROUND}

In this section, we define matrix concepts and patterns, and then we present some functions to evaluate a permuted matrix.

A reorderable matrix $R_{m \times n}$ is a matrix that supports row and column permutations. It may be defined by a non-permutable matrix $N$ and by two permutation vectors: rowOrder and columnOrder, in such a way that

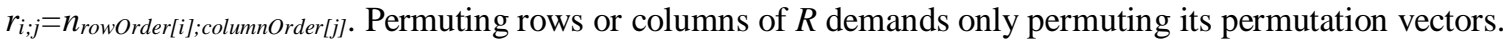

Wilkinson (2005) and Behrisch et al. (2016) present a list of nine patterns that matrices may present. This paper focuses only on Wilkinson's Band and Circumplex patterns. In a matrix with a Band pattern, the closer the cells are to the diagonal, the higher their values. If we consider a matrix as a torus and draw a diagonal band on it, similarly to the Band pattern, we generate the Circumplex pattern. Table 1 exemplifies both patterns. One may configure the bandwidth of Band pattern to a thin or large band. Circumplex has no configuration parameter.

The formal definition of Band and Circumplex patterns (Wilkinson, 2005) begins with the definition of a matrix $X_{n \times p}$ whose cell values are $x_{i, j}=\mathrm{e}^{-t^{2}}+u_{i, j}$. For Band pattern, $t=(j / p-i / n) / b$, where $1 \leq i \leq n$ and $1 \leq j \leq p$; in other words, $t$ measures the distance to the main diagonal, and $b$ defines how sharp is the boundary between low and high values, and also the bandwidth. For Circumplex pattern, $t=\cos (\Pi(j / p-i / n))$. For both patterns, $u_{i ; j}$ is a random error; in our case we replaced it by a salt-and pepper error.

We call pre-Circumplex and pre-Band a matrix whose one or more permutations reveal its Circumplex and Band structure, respectively. We also call Circumplex (or Band) matrix a matrix that obeys the definition of Circumplex (or Band) pattern.

One may use evaluation functions to assess a permuted matrix. Those functions either analyze the data matrix itself, or distance matrices of rows and columns of this matrix (i.e., proximity matrices). Here we focus on the three functions used in this work. Moore neighborhood stress (Niermann, 2005) (Moore stress, for short) measures how similar each cell is in comparison to all its eight neighbors in a matrix. Given a matrix $X_{m, n}$, this measure is defined as stress $=\sum_{\mathrm{i}=1}^{m} \sum_{\mathrm{j}=1}^{n} s(i, j)$, where $s(i, j)=\sum_{k=\max (1, i-1)}^{\min (m, i+1)} \sum_{l=\max (1, j-1)}^{\min (n, j+1)}\left(x_{i, j}-\mathrm{x}_{k, l}\right)^{2}$. Minimal Span Loss Function (MSLF) (Wu et al., 2008) sums the distances of each pair of neighbor rows (or columns) of a matrix. MSLF may use Euclidean Distance as proximity coefficient to measure distance; indeed, one may calculate MSLF directly in a proximity matrix if it is already available. Given a proximity matrix $P_{n, n}$, MSLF is defined as MSLF $=\sum_{i=1}^{n-1} p_{i, i+1}$. Both measures may help to assess permutation quality in a pre-Band matrix, but according to Rocha et al. (2017), they do not fit evaluation needs of a pre-Circumplex matrix. Those authors propose using Circular Correlation as a quality measure. Rocha et al. define this measure as the highest possible absolute Spearman correlation between rows (or columns) of a matrix $A_{m, n}$ and their respective ordering in all possible shifted versions of rows (or columns) in a matrix $B_{m, n}$.

Text citation patterns and data about subjects' preferences for sets of objects are examples where band patterns arise. Circumplex patterns are present in a diversity of psychology phenomena, such as classification of emotions and color perception (Wilkinson, 2005).

Table 1. Examples of synthetic matrices with Band and Circumplex patterns

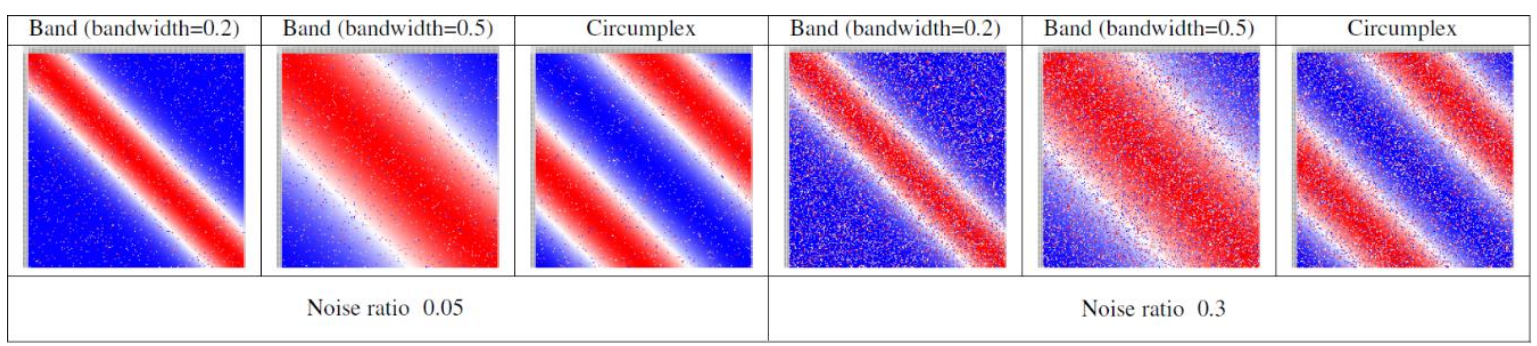

\section{RELATED WORK}

In this section, we classify and exemplify some matrix reordering algorithms (Sect. 3.1). Then we present the research gap related to the use of multidimensional projection approaches for matrix reordering (Sect. 3.2). 


\subsection{Matrix Reordering Approaches}

Behrisch et al. (2016) present seven classes of matrix reordering algorithms. In this work, we highlight the classes of dimensional reduction and spectral algorithms, due to their strong relation to our approach.

Spectral algorithms calculate permutations based on eigenvalues and eigenvectors of proximity matrices of rows (or columns). As an example, Chen (2002) uses a correlation operator $\varphi$ that calculates the correlation matrix of a given matrix (which may be the original matrix or another correlation matrix); he also defines $D$ as the original data matrix, $R^{(1)}=\varphi(D)$, and $R^{(n+1)}=\varphi\left(R^{(n)}\right)$. Chen projects the columns of a correlation matrix as points in a bi-dimensional space defined by the first two eigenvectors of this matrix. When doing this for many values of $n$, he perceived that $R^{(i)}$ may place the projected points into an ellipse, and in an extreme case $\left(\mathrm{R}^{\infty}\right)$ they are projected into two opposite parts of this ellipse. Chen uses both facts to develop approaches for finding a one-dimensional order for points (and their respective columns or rows), and for applying a hierarchical divisive clustering.

Friendly and Kwan (Friendly, 2002) (Friendly \& Kwan, 2003) present another spectral algorithm for reordering a variable-per-variable correlation matrix $C$, which they call "correlation ordering". They use eigen decomposition to calculate the two first eigenvectors of $C: e_{1}$ and $e_{2}$. They define $\alpha[i]$ as the angle of the vector $\left(e_{1}[\mathrm{i}], e_{2}[\mathrm{i}]\right)$ for each row $i$ of $C$. They order the rows of $C$ according to the values of $\alpha$. This circular order is linearized by defining its beginning and ending point where the difference between $\alpha[i]$ and $\alpha[i+1]$ is maximum. $C$ is symmetric, so the order of its columns and rows is the same. Their works exemplify how their method reorders real-world correlation matrices, but they do not use their approach to reorder data matrices.

Dimensional reduction algorithms reduce the number of dimensions $d$ of a dataset to $n$ dimensions, $n<<d$. Outside matrix reordering context, it is usual to define $n=2$, and therefore the dataset is projected into a plane. However, when $n=1$, these algorithms find a linear order of elements, i.e., an order of rows or columns in the reorderable matrix. This approach may use rows' and columns' proximity matrices.

Wilkinson (2005) uses dimensional reduction algorithms for reordering matrices. In his experiments, he compares Singular Value Decomposition (SVD), iterative non-metric MDS (iMDS) (both with $n=1$ ), Locally Preserving Projection (LPP) and a clustering approach. Wilkinson concludes that SVD and iMDS produce the best results for reordering pre-Band and pre-Circumplex matrices (among other types of matrices), in terms of average Spearman Correlation of indexes of rows and columns. However, it is worth noting that Spearman Correlation is not appropriate for Circumplex pattern (see Rocha et al. (2017)).

Apart from those papers, it is worth noting two works from Rehm et al. $(2005,2006)$ that combines MDS and polar coordinates in order to provide dimensional reduction. Both are based on the algorithm $\mathrm{MDS}_{\text {polar }}$, which projects $d$-dimensional points in two dimensions. It preserves the distance between pairs of data points and tries to preserve the angle between those pairs when projecting them to the lower dimension. While they use polar coordinates for projecting points from $d$ to $n$ dimensions, our approach first project points from $d$ to $n$ and then finds a circular ordering of these points by using polar coordinates.

\subsection{Research Gap}

In this section we detail a research gap related to the works of Wilkinson (2005), Friendly (2002), and Friendly \& Kwan (2003). Despite a good evaluation of SVD and iMDS for revealing Band and Circumplex patterns, Wilkinson criticized both methods because they failed on revealing the intrinsic unidimensionality of these patterns. Wilkinson tried to show that this unidimensionality could be captured by using SVD and iMDS with $n=2$ instead of $n=1$. He used two matrices as input: one Band matrix and one Circumplex one. Then he projected their rows and columns in $\mathfrak{R}^{2}$ with SVD and iMDS. He was not interested in the matrix reordering itself at this time, but in showing the resulting projections. The results were four 2D projections (two for SVD, two for iMDS) that we classify into one of the following situations:

1. The proximity of the $d$-dimensional points in the $\mathfrak{R}^{2}$ space defines a circular order (forming an arc or a circle), which corresponds to a good approximation of the correct order of columns (or rows) in the pattern. However, a $1 \mathrm{D}$ projection of the same $d$-dimensional points would not be able to reveal this order. It happens for Circumplex and Band matrices when using SVD, and for Circumplex when using iMDS.

2. The $d$-dimensional points fall within a "football-shaped envelope", and the direction of its major axis reveal a good approximation of the correct order of columns (or rows). Therefore, a 1D projection probably would reveal it. It happens for Band matrices when using iMDS. 
Regarding SVD and Circumplex pattern, Wilkinson writes, "Unfortunately, we cannot detect its unidimensionality unless we convert to polar coordinates, and we cannot know that in advance" (Wilkinson, 2005). Regarding Band pattern, we point out in Section 4 that the projections of its columns (or rows) in 2D also define a circular ordering (given by an arc). Wilkinson states that the projection of the rows or columns of a Band pattern should be unidimensional, but that projection constraints forced the projected points to be in an arc format.

Note that Wilkinson did not use SVD with $n=2$ or iMDS with $n=2$ for reordering matrices. He just indicated that these 2D projections are of interest to observe circular ordering, but he did not reorder matrices based on these projections or evaluate his proposal. Therefore, his idea remains as a hypothesis.

On the other hand, Friendly and Kwan (Friendly, 2002; Friendly \& Kwan, 2003) applied an approach that is similar to Wilkinson's proposal. As already presented in this section, they proposed to order variables of a variable-per-variable correlation matrix according to the angles formed by the first two eigenvectors of this matrix. They present examples of their technique but a formal evaluation is absent. Besides, they cite neither Band nor Circumplex patterns.

In summary, these authors propose the use of algorithms that do a bidimensional projection of rows and columns of a matrix, and uses the circular order of the projected points to sort the rows and columns that they represent. The research gap is that: (1) Wilkinson did not implement and evaluate his bidimensional projection proposal; and (2) Friendly and Kwan did not evaluate their proposal. In order to fill this gap, in this work we propose, implement and evaluate the use of a bidimensional projection in order to generate a circular order to be used for reordering rows and columns of pre-Band and pre-Circumplex matrices.

\section{POLAR SORT}

Based on the research gap, we derived a method for revealing Band and Circumplex patterns in reorderable matrices. Our approach (Polar Sort) analyzes the radial layout of points in 2D projections of rows (or columns) of a pre-Band or pre-Circumplex matrix given as input. First, the method projects the rows (or columns) in $\mathfrak{R}^{2}$. Then it converts the Cartesian coordinate system into a new polar coordinate system whose center is the barycenter of the projected points. Then the method sorts the points by their angular coordinates and replicates this order for rows (or columns) of the matrix.

Instead of using SVD for producing projections with circular order, we opted by using classical MDS (Torgerson, 1952). Table 2 shows the projections for six distinct Band and Circumplex matrices. Color intensity indicates the indexes of rows in the matrix. Each projection helps to note the similarity of order among neighbor points, and that a barycenter of these points would be a possible candidate for the origin of our polar coordinate system. Note that these projections are very similar to those presented by Wilkinson (2005) for SVD outputs and do not produce the undesired football-shaped envelope of the iMDS output for Band.

In order to enhance the output for the case of pre-Band matrices, Polar Sort uses the same strategy of the correlation ordering algorithm (Friendly, 2002). Given the angular ordering of the list of projected points, the method considers this list as circular, and finds the more distant pair of adjacent points in the list. They become the first and the last points of the ordered list that the algorithm will use to reorder rows (or columns). This procedure is needs for Band patterns because of the horseshoe curve of pre-Band projected points. This step does not damage pre-Circumplex ordering.

Regarding asymptotic complexity, Polar Sort algorithm is influenced by the asymptotic complexity of the classical MDS algorithm. Given an input matrix with dimensions $n \times n$, executing the classical MDS algorithm is $\mathrm{O}\left(n^{3}\right)$ (Tzeng et al., 2008). Sorting a list of projected points by angle is $\mathrm{O}(n \log n)$. The remaining steps are linear procedures. Therefore, Polar Sort algorithm is also $\mathrm{O}\left(n^{3}\right)$. A way of improving it is to replace classical MDS by another faster dimensional reduction algorithm that preserves the quality of the output.

We exemplify the results of our algorithm in Table 3. It presents synthetic matrices with dimensions $200 \times 200$ with Band and Circumplex patterns (marked as "Original") and the result of using Polar Sort for reordering a shuffled version of them, i.e., a version with rows and columns randomly permuted. Observe that each pair of original and reordered matrices are quite similar, even for the largest noise ratio presented (0.3). Flipped columns (or rows) do not hamper users to observe Band or Circumplex pattern. Idem for shifted columns (or rows) and Circumplex pattern. 
Table 2. 2D projections of rows of Band and Circumplex matrices with size $200 \times 200$

\begin{tabular}{|l|l|l|l|l|l|}
\hline Band (bandwidth=0.2) & Band (bandwidth=0.5) & Circumplex & Band (bandwidth=0.2) & Band (bandwidth=0.5) & Circumplex \\
\hline & & & \\
\hline
\end{tabular}

Table 3. Examples of synthetic matrices with Band and Circumplex pattern and their shuffled version after reordering

\begin{tabular}{|c|c|c|c|c|c|c|}
\hline \multirow{2}{*}{ Noise ratio } & \multicolumn{2}{|c|}{ Band (bandwidth=0.2) } & \multicolumn{2}{|c|}{ Band (bandwidth=0.5) } & \multicolumn{2}{|c|}{ Circumplex } \\
\hline & Original & Reordered & Original & Reordered & Original & Reordered \\
\hline 0.05 & & & & & & \\
\hline 0.3 & & & & & & \\
\hline & & & & & & \\
\hline
\end{tabular}

\section{EVALUATION}

In this section, we present how we evaluated our method. First, we assess the output quality and execution time of our algorithm and other related reordering algorithms from the literature. Second, we compare its asymptotic complexity to these algorithms. After that, we show Polar Sort applied to real world data.

\subsection{Synthetic Data}

In this section, we compare reordering algorithms from the literature with our proposal. First, we present MRA tool, used for the comparison process. Then we define how we configured our experiment. We also show and discuss our results in the last part of this section.

We used Matrix Analyzer Reordering tool (MRA) (Silva et al., 2014) to compare our method to other relevant reordering methods from the literature. We inform to MRA the data pattern to use for creating the input matrices, a list of pattern configuration parameters, a list of matrix sizes, a list of salt-and-pepper noise ratios, and a list of reordering algorithms to compare on the test. We also inform the evaluation function and the proximity coefficients for comparison. MRA creates a set of $K$ matrices according to the input parameters, shuffles these matrices (in other words, it transforms them in pre-Band and pre-Circumplex, in our case), and uses each reordering algorithm to reorder each shuffled matrix. It uses as shuffling approach the Knuth's unbiased shuffling algorithm (Knuth, 1981). The tool measures the execution time of each reordering algorithm. MRA also evaluates the quality of the output matrices according to the evaluation functions and the proximity coefficients. The tool generates files with time and quality measures per output matrix, together with mean and standard deviation values for each matrix type. MRA also executes paired t-tests that helps to analyze the winning algorithm for a given evaluation function and a set of matrices.

We configured MRA for executing two experiments, one for Band and other for Circumplex patterns. For both experiments, we defined the matrix size as $200 \times 200$; and we used noise ratios $0.01,0.05,0.1,0.3$, and 0.5 . We tested four configurations of the Band pattern, with bandwidth 0.2, 0.5, 1.0, and 1.5. The Circumplex pattern has no configuration to be set. We also defined $K=50$. In total, we tested a set with 1000 Band matrices $(5$ noise ratios $\times 4$ bandwidths $\times 50$ matrices) and with 250 (5 noise ratios $\times 50$ matrices) Circumplex matrices.

We chose the following reordering algorithms to be compared, due to similarity to Polar Sort or to good previous results related to Band or Circumplex patterns (Rocha et al., 2017): Classical MDS ( $n=1$ ), Eigen Decomposition (correlation ordering of Friendly (2002)), SVD (with $n=2$, in order to improve the results), 
SMB+FV+Median (Medina et al., 2016), Entropy Minimization (EM-ordering), and Circumplex Sort. The strings " $(n=1)$ " and " $(n=2)$ " indicate algorithms that projects data into one and two dimensions, respectively.

We defined Moore stress and MSLF (with Euclidean Distance as proximity coefficient) as evaluation functions for the Band experiment. Both functions indicate differences to be reduced as much as possible among neighbour elements (cells, rows, and columns), in order to reveal a Band pattern. We used Circular Correlation as evaluation function for the Circumplex experiment, according to Rocha et al. (2017).

The evaluation results for Band experiment reveal that all the tested algorithms produce outputs with quite similar values of Moore stress and MSLF, so it is difficult to observe a clear winner in most scenarios. Nevertheless, paired t-tests' results show that Polar Sort and EM-ordering win the other algorithms. Besides, the execution time data indicates that in most scenarios Polar Sort and Classical MDS were the fastest algorithms in our tests, with mean execution time around $0.5 \mathrm{~s}$ (exemplified in Figure 1). We also note that EM-ordering had a mean execution time of $1 \mathrm{~s}$ or more in most scenarios.

The evaluation results for Circumplex experiment (exemplified by the circular correlation for rows in Figure 1) show that Polar Sort and Eigen Decomposition produced the highest correlation values and lowest standard deviations in each scenario of this experiment. Regarding execution time, Polar Sort and Classical MDS have the lowest execution times $(\sim 0.6 \mathrm{~s})$, while Eigen Decomposition spent around $1.1 \mathrm{~s}$ in each scenario.

Given these data, we conclude that Polar Sort is very competitive with respect to other algorithms for revealing Circumplex and Band patterns in pre-Circumplex and pre-Band matrices with size $200 \times 200$.

It is worth noting that we executed our experiments as jobs on a high processing cluster, which has 160 cores Power $7553.3 \mathrm{GHz}, 8$ cores Power 720 3.0 GHz, and 24 cores Intel X5650 2.6 GHz.

For the sake of completeness, we list the asymptotic time complexity of the reordering algorithms used at our experiments: Circumplex Sort - $\mathrm{O}\left(n^{2}\right)$ (Rocha et al. 2017); Classical MDS - $\mathrm{O}\left(n^{3}\right)$ (Tzeng et al., 2008), EM-ordering - $\mathrm{O}(n \log n)$ (Djuric \& Vucetic, 2013), Polar Sort - $\mathrm{O}\left(n^{3}\right)$, SMB+FV+Median - $\mathrm{O}\left(n^{2} \alpha\left(n^{2}\right)\right)$ (Medina et al., 2016), Eigen Decomposition $\mathrm{O}\left(n^{3}\right)$ (Dubrulle et al., 1971). We could not find a reliable reference with the asymptotic time complexity of SVD. Note that $\alpha$ is a function that grows very slowly, known as the inverse of Ackermann's function.

Despite having the highest time complexity when compared to most of the analyzed algorithms, the analysis presented at this section indicates that Polar Sort produces high quality outputs in a short time for the evaluated cases. An open question is how to produce such kind of result with a faster time complexity, in order to reduce the expected increasing of time for bigger matrices.

\subsection{Real World Data}

We present two examples of real world data reordered by Polar Sort. The first one is the Iris data set (Fisher, 1936), available at UCI Machine Learning Repository (Dheeru \& Taniskidou, 2017). It has data about 150 flowers of three distinct classes. In this set, one class (Iris setosa) is completely distinct from the other two, which are not linearly separable; so, the set has two natural clusters. First, we created a dissimilarity matrix of flowers, based on Euclidean distance. Then we normalized it and converted it into a similarity matrix.

Figure 2 (left) presents the original matrix (top-left), and the results of reordering it by each algorithm used in our experiments. Red, white and blue stands for high, mean and low similarity values, respectively. Note that the results of Eigen Decomposition, Polar Sort and SVD methods show the expected two red clusters, and present less noise than the other results (see the stress measures below each matrix).

Our second example is Russell's dataset about classification of words (Russell, 1980). A set of 36 volunteers received a list of 28 expressions ("words or phrases that people use to describe their moods, feelings, temporary states, affect, or emotions", as Russell wrote). Each volunteer classified each expression into one of the following eight emotion categories: arousal, contentment, depression, distress, excitement, misery, pleasure, and sleepiness. Russell create an expression-per-category matrix, whose cells are the frequency in which volunteers classified an expression into a given category. He argues that one may use this matrix "to estimate the order of the eight categories". Figure 2 (right) presents a heatmap of this matrix, after shuffling, in order to simulate how the real data would be received by a researcher. The rightmost heatmap is how Polar Sort reorders this shuffled matrix. Note that the answer has a quasi-Circumplex pattern. Also note that Circular Correlation measures for the Polar Sort output, when compared to the original Russell's matrix, are 1.0 for columns and 0.997 for rows, which indicates that this output has the same pattern than the manually ordered matrix. 

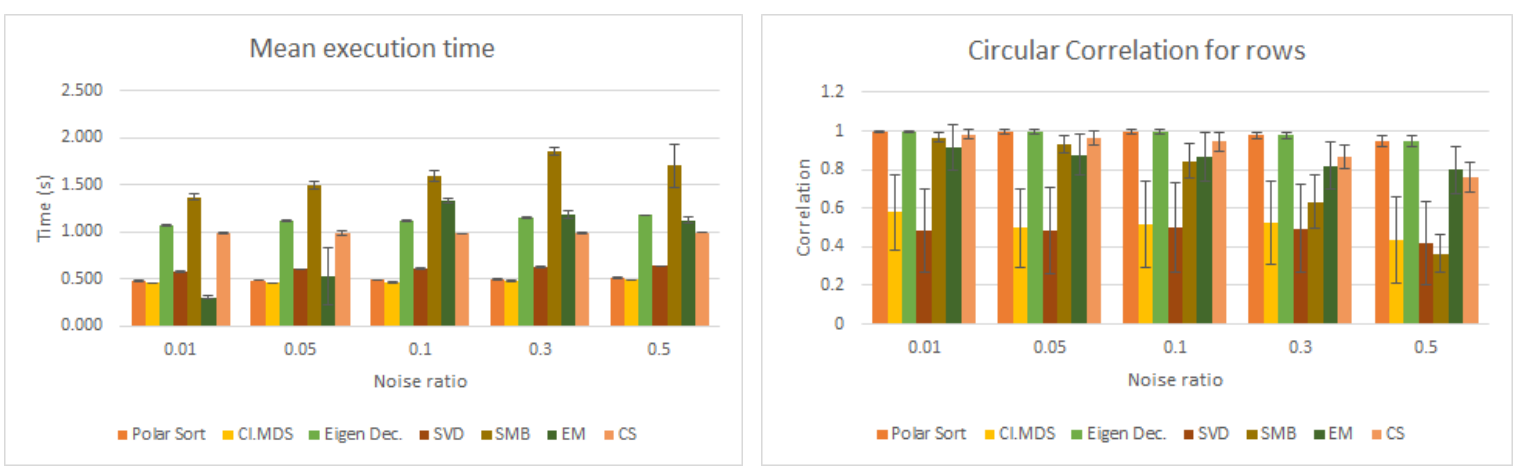

Figure 1. Left: mean execution time for Band experiment $(b=0.5)$. Right: circular correlation for rows in Circumplex experiment.
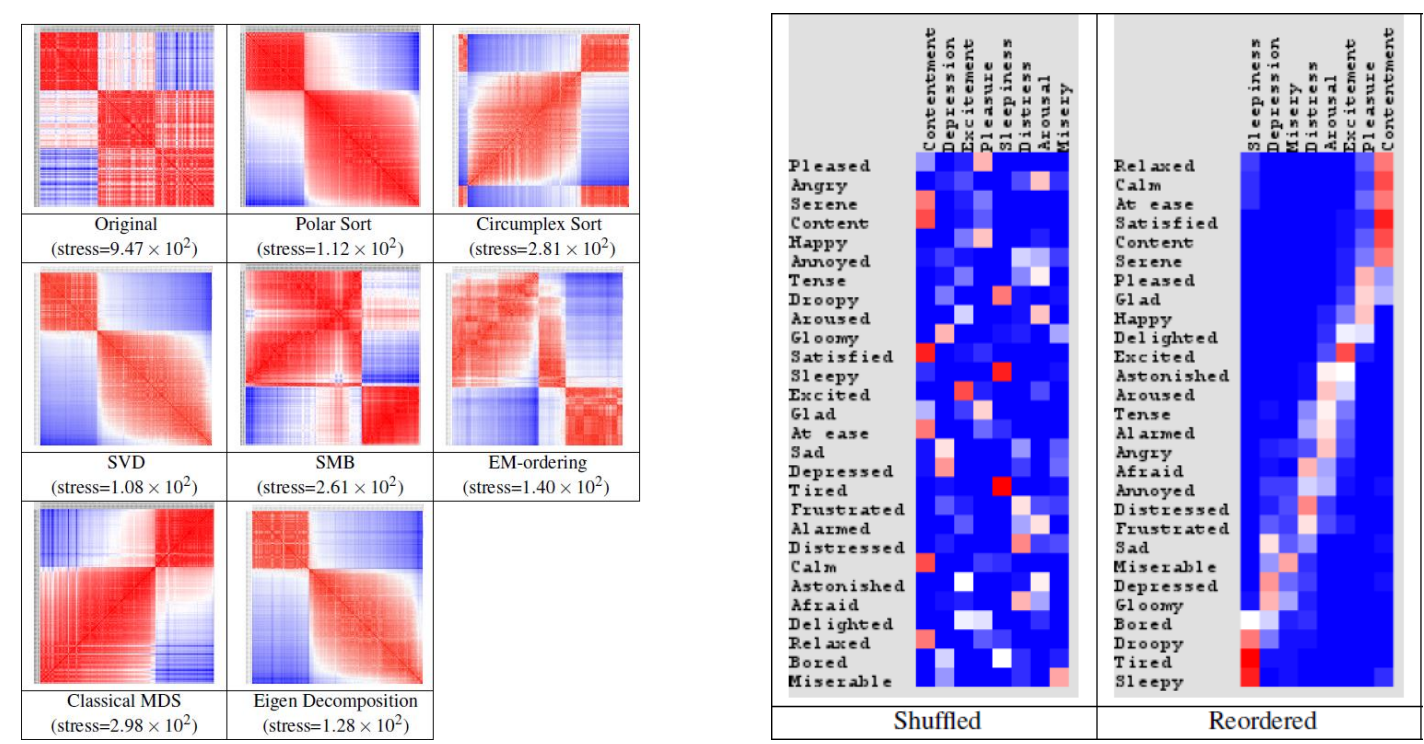

Figure 2. Left: Similarity matrices of Iris dataset, with distinct permutations of rows and columns. Right: Russell's matrix of expressions-per-categories (shuffled and ordered by Polar Sort)

\section{CONCLUSION}

We presented Polar Sort as a fast and high-quality method for reordering both pre-Band and pre-Circumplex matrices. Some reordering methods produce good results only for pre-Band matrices, but not for pre-Circumplex ones; other methods produce good results for both types of matrix, but these methods are time-consuming. Our synthetic experiments revealed that Polar Sort produces high-quality results for both patterns, according to MSLF, Moore stress, and circular correlation, for matrices with size $200 \times 200$. Besides, despite its $\mathrm{O}\left(n^{3}\right)$ time complexity, its mean execution time is as fast as the fastest tested methods: it spent less than $1 \mathrm{~s}$ for reordering a $200 \times 200$ pre-Band or pre-Circumplex matrix, which is an acceptable time for providing a quick answer for users.

A limitation of our work is that one must presume that the matrix to be reordered is pre-Band or pre-Circumplex, given that most of the time there is no information about this classification a priori. Besides, given that our algorithm is $\mathrm{O}\left(n^{3}\right)$, another limitation is regarding scalability. Future works may analyze alternative approaches to classical MDS for dimensional reduction, in order to reduce asymptotic complexity without losing quality of the output matrices. 


\section{ACKNOWLEDGEMENT}

We thank the grant \#2019/04222-4 from São Paulo Research Foundation (FAPESP) and the grant \#2232/19 from FAEPEX/PRP/University of Campinas.

\section{REFERENCES}

Behrisch, M. et al., 2016. Matrix reordering methods for table and network visualization. Computer Graphics Forum, Vol. 35, No. 3, pp. 693-716.

Ben-Dor, A. et al., 2003. Discovering local structure in gene expression data: The order-preserving submatrix problem. Journal of Computational Biology, Vol. 10, No. 3-4, pp. 373-384.

Bar-Joseph, Z. et al., 2001. Fast optimal leaf ordering for hierarchical clustering. Bioinformatics, Vol 17 (Suppl. 1), pp. S22-29.

Chen, C.-H., 2002. Generalized association plots: information visualization via iteratively generated correlation matrices. Statistica Sinica, Vol. 12, pp. 7-29.

Dheeru, D. and Taniskidou, E. K., 2017. UCI machine learning repository. Available at: http://archive.ics.uci.edu/ml [Accessed 01 March 2019].

Dubrulle, A. et al., 1971. The Implicit QL Algorithm. Handbook for Automatic Computation. Springer Berlin Heidelberg, Berlin, Heidelberg, pp. 241-248.

Silva, C. G. et al., 2014. PQR sort: using PQR trees for binary matrix reorganization. Journal of the Brazilian Computer Society, Vol. 20, Issue 1, Article 3.

Silva, C. G. et al., 2017. A fast feature vector approach for revealing simplex and equi-correlation data patterns in reorderable matrices. Information Visualization, Vol. 16, No. 4, pp. 261-274.

Djuric, N. and Vucetic, S., 2013. Efficient visualization of large-scale data tables through reordering and entropy minimization. Proceedings of IEEE 13th International Conference on Data Mining 2013, pp. 121-130.

Fisher, R.,1936. The use of multiple measurements in taxonomic problems. Annual Eugenics, Vol. 7, Part II, pp. 179-188.

Friendly, M. and Kwan, E., 2003. Effect ordering for data displays. Computational Statistics and Data Analysis, Vol. 43, No. 4, pp. 509-539.

Friendly, M., 2002. Corrgrams. The American Statistician, Vol. 56, No. 4, pp. 316-324.

Knuth, D. E., 1981. The Art of Computer Programming, Vol. 2. Addison-Wesley.

Lenstra, J. K., 1974. Clustering a data array and the traveling salesman problem. Operations Research, Vol. 22, No. 2, pp. 413-414.

Medina, B. F. et al., 2016. Smoothed Multiple Binarization - Using PQR tree, smoothing, feature vectors and thresholding for matrix reordering. Proceedings of $20^{\text {th }}$ International Conference Information Visualisation (IV), pp. 88-93.

Mäkinen, E. and Siirtola, H., 2000. Reordering the reorderable matrix as an algorithmic problem. Theory and Application of Diagrams. Springer, pp. 453-468.

Niermann, S., 2005. Optimizing the ordering of tables with evolutionary computation. The American Statistician, Vol. 59, No. 1, pp. 41-46.

Rehm, F. et al., 2005. MDS polar: A new approach for dimension reduction to visualize high dimensional data. Advances in Intelligent Data Analysis, LNCS, Vol. 3646, pp. 316-327.

Rehm, F. et al., 2006: Polarmap - efficient visualization of high dimensional data. Proceedings of the 10th International Conference on Information Visualisation (IV'06), pp. 731-740.

Rocha, M. M. N. et al., 2017. Circumplex sort: a two-phase method for revealing circumplex data patterns in reorderable matrices. Proceedings of International Conferences Computer Graphics, Visualization, Computer Vision and Image Processing 2017 and Big Data Analytics, Data Mining and Computational Intelligence 2017, pp. 181-188.

Russell, J., 1980. A circumplex model of affect. Journal of Personality and Social Psychology, Vol. 39, No. 6, pp. 1161-1178.

Tzeng, J. et al., 2008. Multidimensional scaling for large genomic data sets. BMC Bioinformatics, Vol. 9, Article 179.

Torgerson, W. S., 1952. Multidimensional scaling: I. theory and method. Psychometrika, Vol. 17, No. 4, pp. $401-419$.

Wilkinson, L., 2005. The Grammar of Graphics. Springer Science \& Business Media.

Wu, H.-M. et al., 2008. Matrix Visualization. Handbook of Data Visualization. Springer, pp. 681-708. 